\title{
Satisfying End-to-End Quality of Service Requirements in Mobile Packet Networks
}

\author{
Patrick Fiati \\ Department of Electrical, Electronic Engineering, \\ Kwame Nkrumah University of Science and Technology, \\ Kumasi, Ghana
}

\begin{abstract}
Internet service providers face a daunting challenge in provisioning network resources, due to the rapid growth of the Internet and wide fluctuations in the underlying traffic patterns. The ability of dynamic routing to circumvent congested links and improve application performance makes it Ia valuable traffic engineering tool. However, deployment of load-sensitive routing is hampered by the overheads imposed by link-state update propagation, path selection, and signaling. Under reasonable protocol and computational overheads, traditional approaches to load-sensitive routing of IP traffic are ineffective, and can introduce significant route flapping, since paths are selected based on out-of-date linkstate information. Although stability is improved by performing load-sensitive routing at the flow level, flapping still occurs, because most IP flows have a short duration relative to the desired frequency of link-state updates. To address the efficiency and stability challenges of loadsensitive routing, we introduce a new hybrid approach that performs dynamic routing of long-lived flows, while forwarding shortlived flows on static preprovisioned paths. By relating the detection of long-lived flows to the timescale of link-state update messages in the routing protocol, route stability is considerably improved
\end{abstract}

\section{Keywords}

OSPF, IS-IS, RIP, MPLS, UMTS

\section{INTRODUCTION}

Traffic engineering of large IP backbone networks has become a critical issue in recent years, due to the unparalleled growth of the Internet and the increasing demand for predictable communication performance [1]. Ideally, an Internet Service Provider (ISP) optimizes the utilization of network resources by provisioning of backbone routes based on the load between the edge routers. However, the volume of traffic between particular points in the network can fluctuate widely over time, due to variations in user demand and changes in the network configuration, including failures or reconfigurations in the networks of other service providers. Currently, network providers must resort to coarse timescale measurements to detect network performance problems, or may even depend on complaints from their customers to realize that the network requires reconfiguration [2]. Detection may be followed by a lengthy diagnosis process to discover what caused the shift in traffic. Finally, providers must manually adjust the network configuration, typically redirecting traffic by altering the underlying routes. These traffic engineering challenges have spurred renewed interest in dynamic routing as a network-management tool, rather than as a method for providing quality-of-service (QoS) guarantees. By selecting paths that circumvent congested links, dynamic routing can balance network load and improve application performance. Despite these potential benefits, however, most backbone networks still employ static routing (e.g., based on routing protocols such as OSPF and IS-IS) because techniques for load-sensitive routing often lead to route flapping and excessive control traffic overheads. Early attempts in the ARPANET to route based on dynamic link metrics resulted in dramatic fluctuations in link load over time. Routing packets based on out-of-date link-state information caused flapping, where a large amount of traffic would travel to seemingly under-utilized links. These links would become overloaded, causing future packets to route to a different set of links, which would then become overloaded. Improvements in the definition of the link metrics reduced the likelihood of oscillations, but designing stable schemes for load-sensitive routing is fundamentally difficult in packetbased networks like the Internet. With the evolution toward integrated services in IP networks, recent research focuses on load-sensitive routing of flows or connections, instead of individual packets. For example, a flow could correspond to a single TCP or UDP session, all IP traffic between a particular source-destination pair, or even coarser levels of aggregation. In particular, several QoS routing schemes have been proposed to select paths based on network load, as well as application traffic characteristics and performance requirements.

Dynamic routing of flows should be more stable than selecting paths at the packet level, since the load on each link should fluctuate more slowly, relative to the time between updates of link-state information. Also defining network load in terms of reserved bandwidth and buffer space, rather than measured utilization, should enhance stability.

However, QoS-routing protocols impose a significant bandwidth and processing load on the network, since each router must maintain its own view of the available link resources, distribute link-state information to other routers, and compute and establish routes for new flows. The protocol and computational overheads can be significant in large backbone networks. Since most TCP/UDP transfers consist of just a handful of packets, load-sensitive routing of all flows would require frequent propagation of link-state metrics and recomputation of routes to avoid the same instability problems that arise in dynamic routing at the packet level. We address this problem by proposing and evaluating a hybrid routing scheme that exploits the variability of IP flow durations to avoid the undesirable effects of traditional approaches to dynamic routing.

While most Internet flows are short-lived, the majority of the packets and bytes belong to long-lived flows, and this property persists across several levels of aggregation. Although this inherent variability of Internet traffic sometimes complicates the provisioning of network bandwidth and buffer resources, heavy-tailed flow-size distributions can be exploited to reduce the overheads of certain control 
mechanisms. Most notably in the networking context, variability in flow duration has been the basis of several techniques that reduce router forwarding overheads by establishing hardware switching paths for long-lived flows. These schemes classify arriving packets into flows and apply a trigger (e.g., arrival of some number of packets within a certain time interval) to detect long-lived flows. Then, the router dynamically establishes a shortcut connection that carries the remaining packets of the flow. The shortcut terminates if no packets arrive during a predetermined timeout period (e.g., 60 seconds). Several measurement-based studies have demonstrated that it is possible to limit the setup rate and the number of simultaneous shortcut connections, while forwarding a large fraction of packets on shortcuts. We focus on dynamic routing as a traffic engineering technique that reacts to fluctuations in network load, rather than as a way to provide explicit performance guarantees.

\subsection{Questionnaire}

(Q1) In your opinion, which of the following do you think is the best routing protocol in data packet networks such as $3.5 \mathrm{G}$ networks.
(a) MPLS
(b) OSPF
(c) IS- IS (d) RIP

(Q2) Your choice in question (1) is mostly deployed in modern ISPs and 3.5G networks.
(a) YES
(b) NO don't know

(Q3) Your choice in question (1) has matured a lot and it's a stable technology.
(a) YES
(b) NO don't know

(Q4) Your choice in question (1) is based on IP and the internet is based on IP.
(a) YES
(b) NO don't know

(Q5) The future of your choice in question (1) is ensured for quite a while to come.
(a) YES
(b) $\mathrm{NO}$ don't know

(Q6) Your choice in question (1) can circumvent route flapping and network congestion.
(a) YES
(b) NO don't know

(Q7) Your choice in question (1) can transport IPv4, IPv6, Ethernet, High Level Data Link Control (HDLC) and other Layer 2 technologies.
(a) YES
(b) NO don't know

(Q8) Your choice in question (1) outperforms traditional static and dynamic algorithms.
(a) YES
(b) NO don't know

(Q9) When there are multiple paths in routing, deploying your choice in question (1) is the best option in selecting the shortest path.
(a) YES
(b) $\mathrm{NO}$
don't know

(c) I

If No, give a reason METHODOLOGY

\subsection{Overview}

Surveys are to be conducted in order to collect primary and secondary data, analyse them and determine the best routing protocol to satisfy the end to end quality of service requirement in mobile packet networks such as $3 / 3.5 \mathrm{G}$ networks. Secondary data will focus on the advantages and disadvantages as well as the reliability of possible candidate routing protocols. Primary data are to be collected by surveying views of Senior Managers of telecommunication operators in line with this topic. Specifically, a total of 50 respondents are to be randomly selected from MTN to make up the sample. The survey questionnaire is to be structured in the Likert format. Data gathered from this research instrument is then to be analysed and interpreted. Along with primary data, secondary data are to be gathered in the form of published articles and literatures from the Institute of Electrical and Electronic Engineering (IEEE) journals and Association of Computing Machines (ACM) website to support the survey results.

\subsection{Results, Discussion and Analysis}

The questionnaire was designed to provide to two analytical approaches. Firstly descriptive statistics were used to analyse data from closed questions which consisted predominantly of nominal level codes. Ordinal level data was obtained from technical managers comments regarding routing protocols, indicating a rank order without quantifiable increments. These were categorised without inferring a quantitative meaning [3]. Charts and tables where appropriate illustrate these data, and descriptive statistics were judged to be the most appropriate for this research due to its size - generalising to a target population through inferential statistics was not appropriate.

Secondly, the qualitative free text was analysed for thematic content and used to evaluate open ended questions, which involves looking for commonalities among participants and assessing relationships within data. Therefore common reasons for selecting the best routing protocol in data network were extracted and compared.

Each response on the questionnaire was coded (where possible) and entered into Microsoft Excel. When the computerised data set was complete, each question was examined separately and frequency of responses displayed in a table. Appropriate information was presented in graph form and cross-tabulated to aid understanding and interpretation [4]. The open ended (qualitative) responses were categorised and responses entered into a spreadsheet in an attempt to categorise responses. Contingency tables (cross tabulation of two ordinal or nominal level variables) were used to identify potential relationships between answers.

\subsection{Presentation of Results and Discussion}

Figure 4.1 shows that 40 technical managers chose MPLS as the best routing protocol in data packet networks such as 3.5G, 5 technical managers chose OSPF, 3 chose IS-IS and 2 chose RIP. 


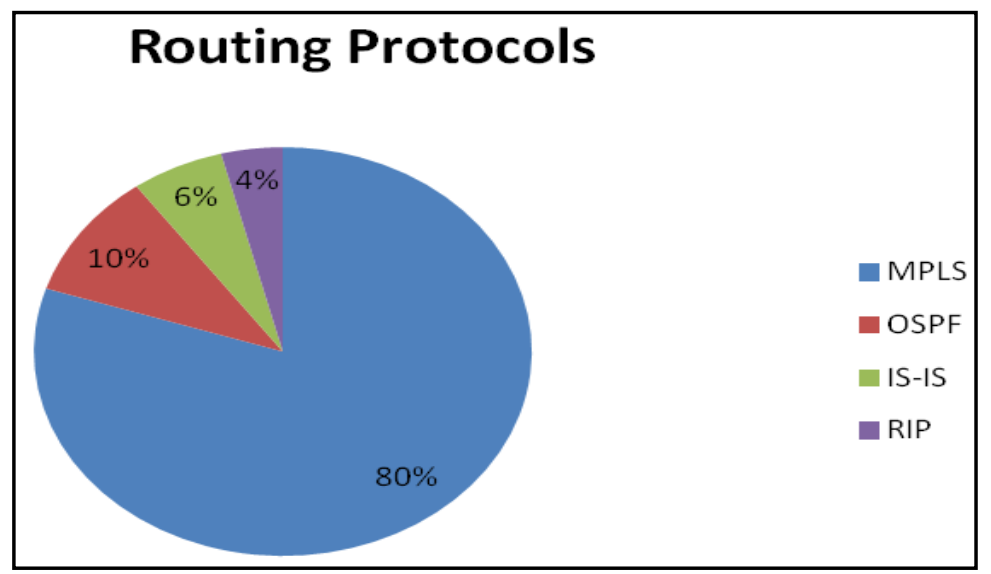

Figure 4.1 (Q1): The best routing protocol used in data networks such as 3.5G

Figure 4.2 shows the respondents answering whether their answer in (Q1) is the most deployed in modern ISPs and 3.5G networks. Majority of the technical managers answered YES then followed by I don't know and very few answered NO.

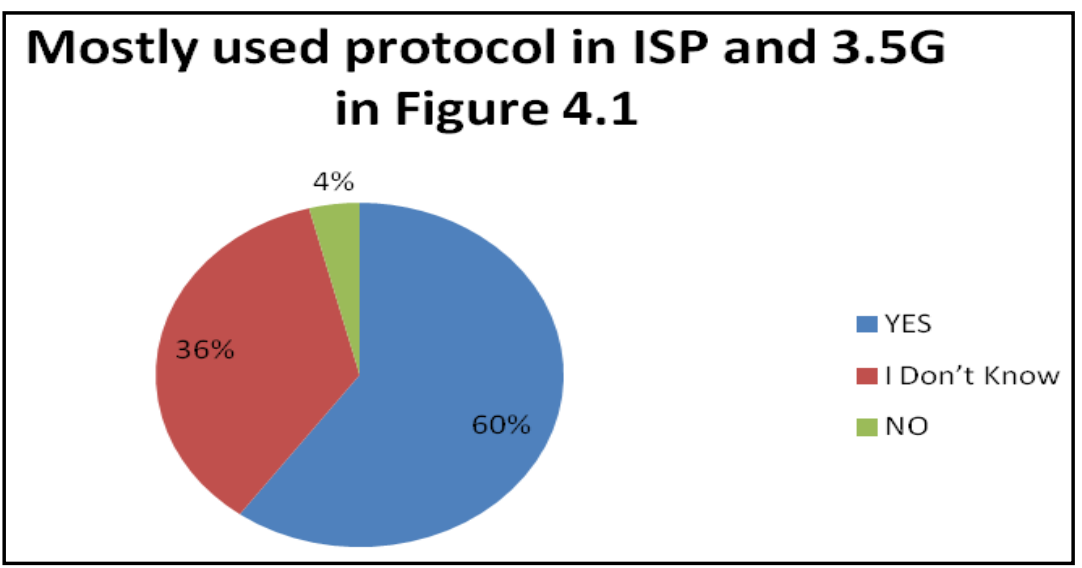

Figure 4.2 (Q2): Your choice (Q1) is mostly deployed in modern ISPs and 3.5G networks.

Figure 4.3 shows whether the answered chosen in (Q1) has matured a lot and it is a stable technology. Majority of the respondents $(70 \%)$ or 35 out of 50 believe their answer to best protocol used in data packet network has really matured and has been tested for several times and it is a stable technology that can be relied on. $28 \%$ answered NO indicating the answer to Q1 is not matured and it not a stable technology. 2\% answered I don't know indicating they either don't know or they are not very sure whether their answer to Q1 has matured and it's a stable technology.

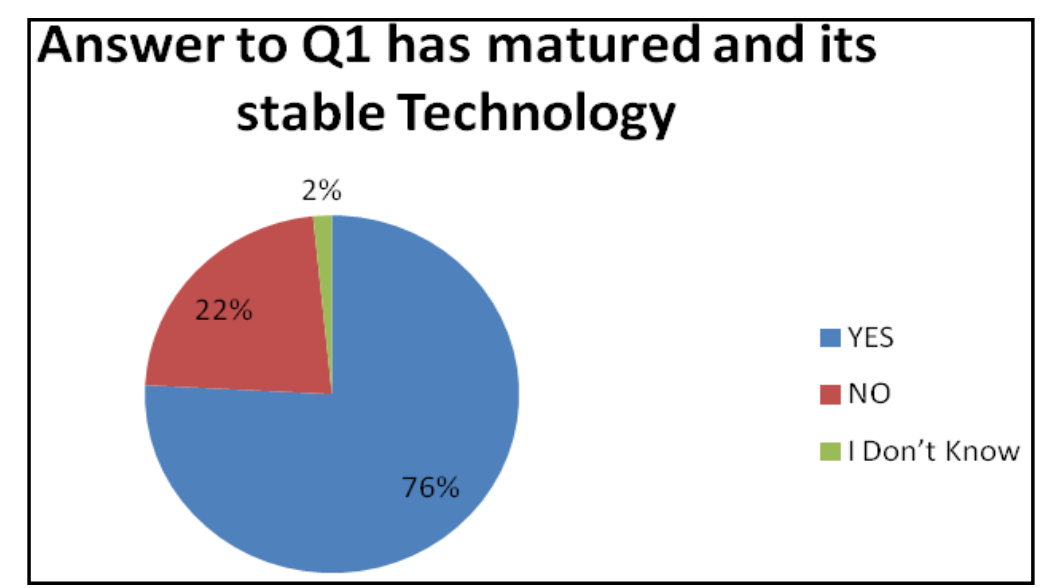

Figure 4.3 (Q3): Your choice in (Q1) has matured a lot and it's a stable technology.

Figure 4.4 shows whether the answer chosen in (Q1) is based on Internet Protocol (IP). 47 out of 50 respondents answered YES representing 94\%, those who chose I don't know were 3 out of 50 representing $6 \%$, Non of the respondents chose NO indicating $0 \%$. 


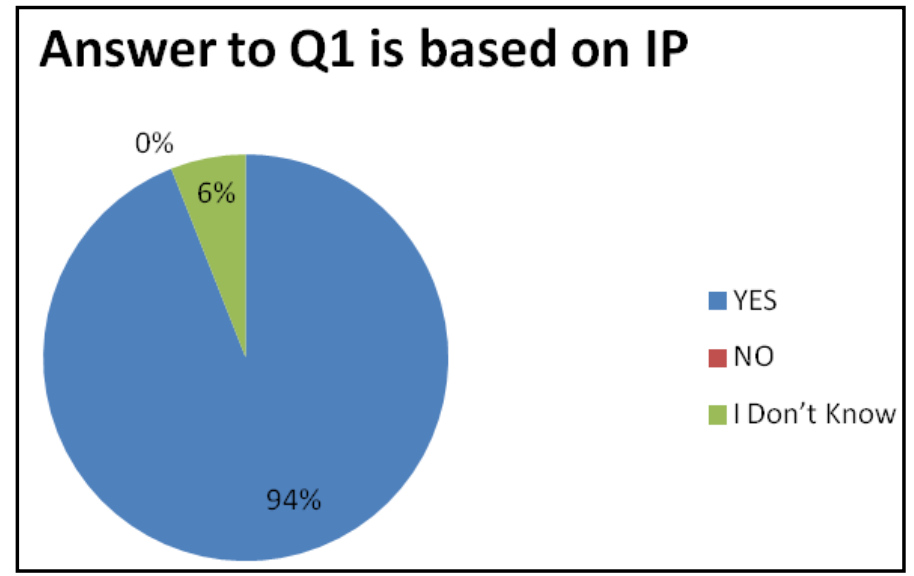

Figure 4.4 (Q4): Your choice (Q1) is based on IP and the internet is based on IP.

Figure 4.5 shows whether the future of the answer to Q1 is ensured for quite a while to come. This was to investigate the respondents view for the future of their answer in Q1, whether they believe will become a well acceptable protocol in the future ahead of the rest. Majority answered YES representing
$86 \%$ (43 out of 50 respondents) indicating they are sure their of the best routing protocol for data packet network will dominate in the future. $12 \%$ answered I don't know and $2 \%$ answered NO meaning their answer to Q1 is not future guaranteed.

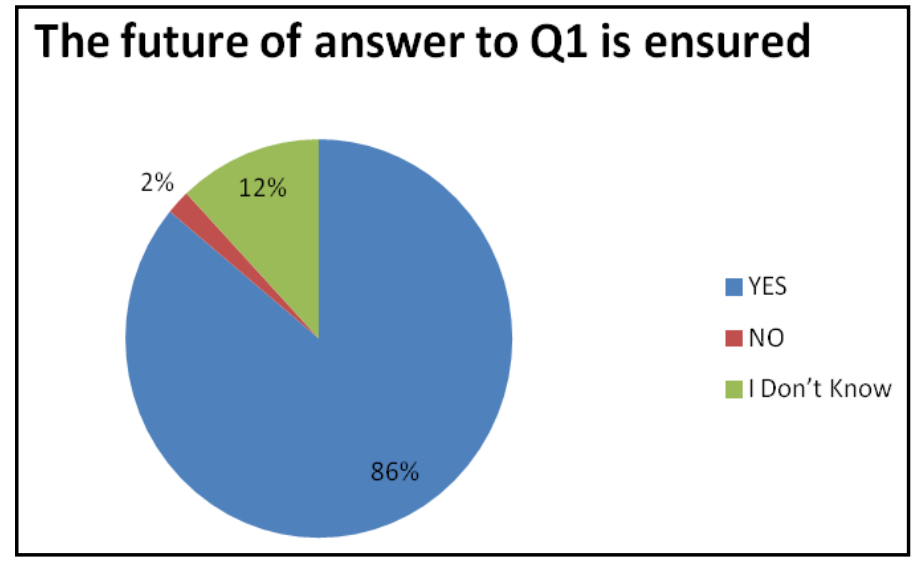

Figure 4.5 (Q5): The future of your choice in (Q1) is ensured for quite a while to come and it is reliable.

Figure 4.6 Illustrates whether the answer to (Q1) can avoid or circumvent route flapping and network congestion. Majority of the respondents chose YES representing 98\% (49 out of 50 respondents) indicating most of the respondents believe that their choice of routing protocol can avoid route flapping and network congestion. $2 \%$ answered I don't know while $0 \%$ answered NO.

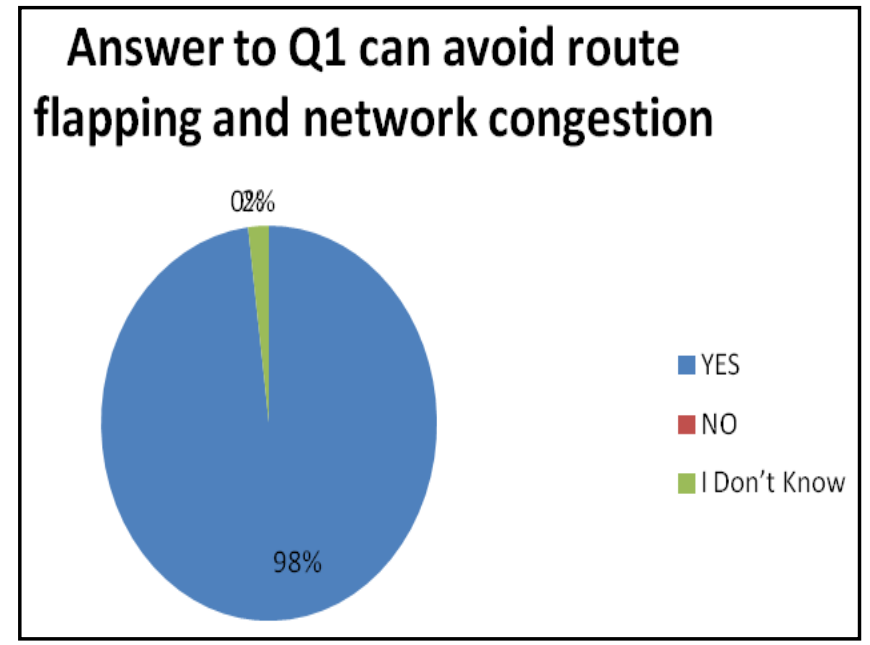

Figure 4.6 (Q6): Your choice in (Q1) can circumvent route flapping and network congestion. 
Figure 4.7 show whether the choice in Q1 can transport IPv4, IPv6, Ethernet, High Level Data Link Control (HLDLC) and other layer 2 technologies. 44 out of 50 answered YES (88\%) their choice of the best routing protocol in data packet network can transport IPv4, IPv6, Ethernet, HLDLC and other layer 2 technologies. 4 out 50 respondents $(8 \%)$ answered I don't know whiles 2 out of 50 respondents answered NO which represents $(4 \%)$.

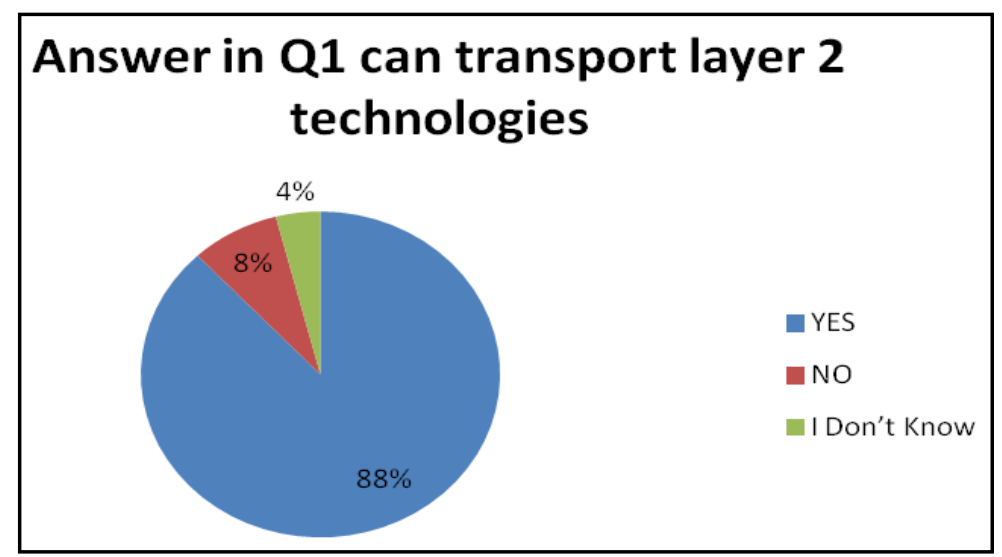

Figure 4.7 (Q7): Your choice in (Q1) can transport IPv4, IPv6, Ethernet, High Level Data Link Control (HDLC) and other Layer 2 technologies.

Figure 4.8 demonstrates the choice of answer in Q1 outperforms traditional static and dynamic algorithms. 50 out 50 respondents answered YES representing a $100 \%$ total agreement. Nobody answered for both NO and I don't know which represents $0 \%$ each.

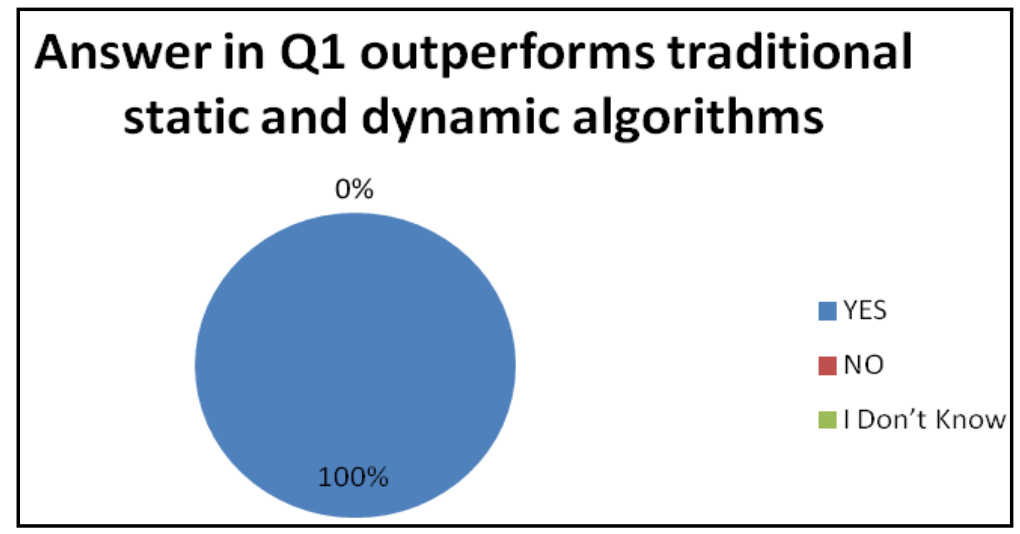

Figure 4.8 (Q8): Your choice in (Q1) outperforms traditional static and dynamic algorithms

Figure 4.9 shows whether when there are multiple paths in routing, deploying your choice in $(\mathrm{Q} 1)$ is the best option in selecting the shortest path. $70 \%$ representing 35 out 50 respondents answered YES followed by $24 \%$ (12 out of 50 respondents) answered $\mathrm{NO}$ and then $6 \%$ (3 out of 50) also answered I don't know.

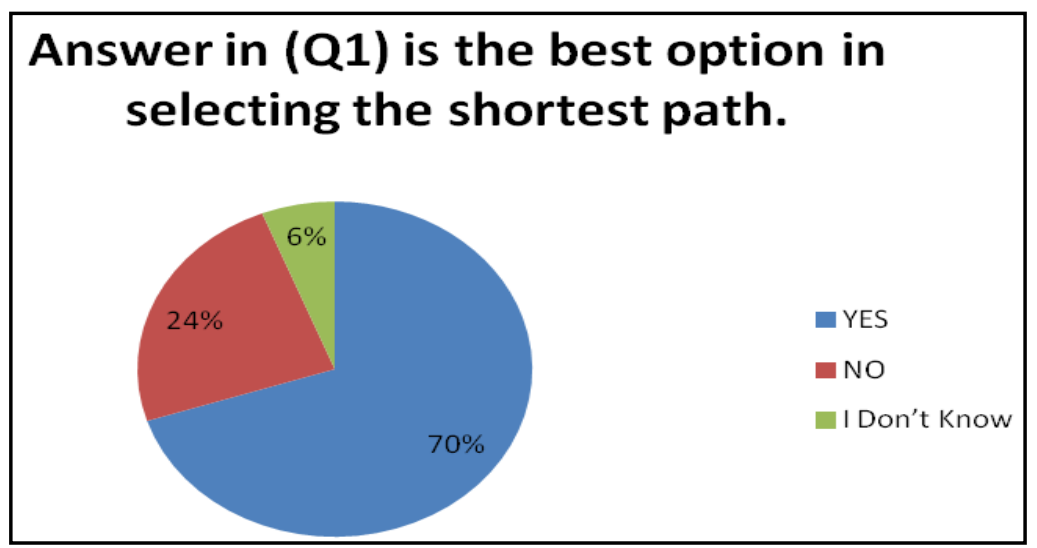

Figure 4.9 (Q9): When there are multiple paths in routing, deploying your choice in (Q1) is the best option in selecting the shortest path. 


\subsection{Result Analysis}

Results from the respondents indicated that MPLS as their preferred protocol. Many researchers have done a lot of works in the areas of MPLS routing protocols where they came with results that corresponds with that of the respondents in this study. Some studies done by S. Jha and M. Hassan in 2002 [5] showed that MPLS is a label-forwarding scheme which provides a better solution to address the problems faced by present-day networks-speed, scalability, quality-of-service (QoS) management, and traffic engineering. They also showed that, MPLS has emerged as an elegant solution to meet the bandwidth-management and service requirements for next generation IP-based backbone networks. Fabino M. et al. [6] in their studies gave the advantages of using MPLS which makes them to dominate in mobile, wireless communication networks.

The purpose of traffic engineering (TE) is to enhance network utilization and to improve the architecture of a network in a systematic way, so that the network becomes robust, adaptive and easy to operate. MPLS has extended routing capabilities that efficiently controls the network traffic by removing congestion and spreading the load over different links. Different route selection algorithm based on MPLS frame work is provided in [7-8]. This corresponds to the results from the respondents where they were asked whether their choice of protocol can circumvent route flapping and network congestion. Majority of them (98\%) answered YES. The results also indicated that, majority of the respondents (88\%) agreed that their choice of protocol can transport IPv4, IPv6, Ethernet, High Level Data Link Control (HDLC) and other Layer 2 technologies. Some research have also been done to show interoperability and performance evaluation between IPv4 and IPv6 with MPLS. Yunos R. et al.[9] conducted research on performance evaluation between IPv4 and IPv6 with Linux MPLS tunnel. MPLS Linux tunneling is used to transport IPv6 data stream over IPv4 network for interoperable IPv4 and IPv6 deployment. The performance metrics such as jitter, datagram/packet loss and bandwidth were measured in both TCP and UPD traffic flow. The experiment test-bed was constructed using virtual machine tool for simulating the IPv4, IPv6, and the MPLS tunneling mechanism. Their study revealed that IPv6 offered better performance than IPv4 in almost all of the testing except in TCP transfer. In addition, MPLS tunnel improved performance in packet transfer for TCP transfer, UDP transfer and bandwidth testing. MPLS which combines the flexibility of Layer3 routing with Layer2 switching is being widely developed. Unfortunately, existing MPLS specifications can only support unicast well, but lack of supporting multicast. The main difficulty in MPLS supporting multicast is how to bind labels to multicast FEC. Zhongshang Zhang et al.[10] in their studies presented a new mechanism for MPLS supporting IP multicast routing protocol. In their proposed mechanism, label distribution is triggered by traffic and not by control messages. This mechanism makes the network's scalability and dynamic property better than the mechanism whose label distribution is triggered by control messages.

Tran Cong et al. [11] also focused their study on interoperability between mobile IPv4 and mobile IPv6. For better QoS services and how their proposal can be applied for large network on Internet, they developed their solution on an MPLS network that is now used for QoS and high-speed core network. In the case of protocol reliability, maturity and stability, $76 \%$ answered YES whiles $22 \%$ answered NO and $2 \%$ answered I don't know for their choice of protocol maturity and stability. For reliability, $86 \%$ answered YES $12 \%$ answered NO and $2 \%$ answered I don't know.

Chen and Oh in 1999 [12] performed some research to examine the reliability of multiprotocol label switching (MPLS) .MPLS is a convergence of various implementations of IP switching using ATM-like "label swapping" to speed up packet forwarding without changes to existing IP routing protocols. An important practical issue is the capability to recover quickly from faults. In their work, they examined distributed methods for fast fault recovery using modified label distribution protocol messages [13]. To maintain and verify service continuity, methods are proposed for traffic and performance monitoring. All these studies showed some level of agreement with our studies.

\section{CONCLUSION}

Internet Service Providers or $3.5 \mathrm{G}$ networks face difficult challenges in engineering large backbone networks due to wide fluctuations in the underlying traffic and increasing user demands for predictable communication performance. Dynamic routing can play an important role in traffic engineering of ISP networks or $3.5 \mathrm{G}$ networks, if selecting routes based on load can be made both stable and efficient.

The purpose of traffic engineering (TE) is to enhance network utilization and to improve the architecture of a network in a systematic way, so that the network becomes robust, adaptive and easy to operate. The routing protocols OSPF, IS-IS, RIP and MPLS have been critically studied to determine the best for $3.5 \mathrm{G}$ networks.

In addition to this a survey was conducted to get the views of Senior Managers of telcos such as MTN. The results of this exercise suggest that MPLS is the protocol of the future. This is because it satisfies end-to-end QoS requirements in 3.5G mobile packet networks.

MPLS outperforms traditional static and dynamic routing algorithm such as OSPF, IS-IS and RIP. When there are multiple paths in routing, deploying MPLS is the best option in selecting the shortest path. MPLS can circumvent route flapping and network congestion. In addition, it shows that MPLS is robust to inaccuracies in network provisioning and shifts in the offered traffic. The MPLS labels are advertised between routers so that they can build a label-to-label mapping. These labels are attached to the IP packets, enabling the routers to forward the traffic by looking at the label and not the destination IP address. The packets are forwarded by label switching instead of by IP switching.

In conclusion, given the fact that MPLS is based on IP, and the internet is based on IP technology, it seems that the future of MPLS is ensured for quite a while to come. It is therefore recommended that MPLS is the best routing protocol for most 3.5G networks and it is suggested that its Operations and Maintenance as a routing protocol can be studied for future works.

\subsection{Future Work}

Currently there are no specific mechanisms proposed to address requirements for user and data plane Operations and Maintenance (OAM) for Multi-Protocol Label Switching (MPLS). The main goal is to identify commonly applicable set of requirements for MPLS OAM. Specifically, a set of requirements that apply to most common set of MPLS networks deployed by service providers. These requirements can then be used as a base for network management tool development and to guide the evolution of specified tools, as 
well as the specification of OAM functions that are intrinsic to protocols used in MPLS networks.

\section{REFERENCES}

[1] University of Michigan, Real-Time Computing Laboratory, Department of EECS; AT\&T LabsResearch, Network Mathematics Research, Networking and Distributed System; Load- Sensitive Routing of Long-Lived IP flows.

[2] G. Rutka, Some Aspects of Traffic Analysis for internet Traffic Prediction, Faculty of Electronics and Telecommunication, Riga Technical University, Riga, Azenes str.12-317, LV-1048 phone:+371 29627600, email: gundega.rutka@rtu.lv ISSN 1392-1215 2009 No.5(93).

[3] Polit D. F. and Hungler B.P. (1995) "Nursing Research: Principles and Methods.

[4] Walsh M. (2001) Research Made Real. Cheltenham. Nelson Thornes Ltd.

[5] Jha S. and Hassan M. (2002) "Engineering Internet QoS", Artech House.

[6] Chiussi, F. M. Khotimsky, D. A. Krishnan, S. (sept. 2002) "Mobility Management in 3G All IP Networks", IEEE Communication Magazine, Lucent Technologies.
[7] Youngseek, L. Yongho, S. Yanghee C. Changhoon, K. (2002) "A Constrained Multipath Traffic Engineering Scheme for MPLS Networks", Proc. Of IEEE Inst. Conf. ICC New York.

[8] Vasu, J. Shahram, L. "An Overview of MPLS and Constraint Based Routing” www.unlv.edu.

[9] Shyam, S. Venkatessan M."Alternate Path Routing Algorithm Engineering", www.ee.edu.

[10] Yunos, R., Noor, N. M., Ahmad, S. A., (2010) "Performance Evaluation Between IPv4 and IPv6 on MPLS Linux Platform" Information Retrieval and Knowledge Management CAMP) pp. 204-208.

[11] Zhongshan, Z. Keping L., Wendong, W., Shiduan, C. (2000) "The New Mechanism for MPLS Supporting IP Multicast", IEEE Circuits and Systems Asia-Pacific Conf. pp. 247

[12] Tran, C. H., Nguyen, N. C., Nguyen D. T., Truong D. H. (2007), " Interoperability between Mobile IPv4 and Mobile IPv6 based on MPLS Core Network", IEEE Advanced Communication Technology, The $9^{\text {th }}$ international conference vol. 2 pp. 1187.

[13] Chen, T. M., Oh, T. H. (1999), "Reliable Services in MPLS", Communications Magazine, IEEE. Southern Methodist Univ. Dallas, TX pp. 58 